\title{
MODELING AND SIMULATION OF ARCHITECTURED IRON-BASED SMA MATERIALS
}

\author{
Wael Zaki \\ Khalifa University \\ 127788 Abu Dhabi, UAE \\ Email: wael.zaki@kustar.ac.ae
}

\author{
Cheikh Cissé \\ Khalifa University \\ 127788 Abu Dhabi, UAE \\ Email: cheikh.cisse@kustar.ac.ae
}

\author{
Tarak Ben Zineb \\ Université de Lorraine \\ LEMTA, UMR 7563 \\ F-54500, Vandoeuvre-lès-Nancy, France \\ Email: tarak.ben-zineb@univ-lorraine.fr
}

\section{ABSTRACT}

The paper presents results of finite element analysis of architectured iron-based shape memory alloy (SMA) samples consisting of bulk SMA and void combined to different proportions and according to different geometric patterns. The finite element simulation uses a constitutive model for iron-based SMAs that was recently developed by the authors in order to account for the behavior of the bulk material. The simulation of the architectured SMA is then carried out using a unit cell method to simplify calculations and reduce computation time. For each unit cell, periodic boundary conditions are assumed and enforced. The validity of this assumption is demonstrated by comparing the average behavior of one unit cell to that of a considerably larger sample comprising multiple such cells. The averaging procedure used is implemented numerically, by calculating volume averages of mechanical fields such as stress and strain over each finite element model considered as a combination of mesh elements.

\section{INTRODUCTION}

Shape memory alloys (SMAs) are known for their ability to experience severe deformation that can be recovered by heating or mechanical unloading. This ability is a manifestation of reversible phase transformation between two solid phases: austenite and martensite, characterized by different degrees of crystallographic symmetry. By far the most common SMA is an alloy consisting predominantly of nickel and titanium, which nonetheless comes at higher cost and presents significant challenges in manufacturing compared to other SMAs. Consequently, with few exceptions, NiTi products are usually found in the form of relatively thin wires, bars and plates. In cases where cost and manufacturability are a primary concern, the more recently developed iron-based SMAs (Fe-SMAs) present an interesting alternative. Some of these Fe-SMAs such as Fe-Pt and Fe-Pd alloys are capable of thermoelastic deformation with a high shape memory effect (1), whereas others, including Fe-Mn-Si and Fe$\mathrm{Ni}-\mathrm{C}$ present larger thermal hysteresis and limited shape memory (2). In all cases, Fe-SMAs offer better machinability and manufacturability compared to NiTi $(3 ; 4)$. Despite their strong potential for engineering applications, constitutive models for $\mathrm{Fe}$ SMAs, especially for the case of multiaxial loading, have only recently been developed $(5 ; 6)$. In contrast, models for NiTi are by now well developed $(7 ; 8)$.

In the present work, the behavior of architectured Fe-SMAs is investigated by means of finite element analysis of cells of different geometries, assumed to possess a periodic structure. The modeling framework is similar to the one described in $(9 ; 10)$ and previously used in modeling NiTi SMAs $(11 ; 12)$. The numerical analysis in this case is carried out on an elementary unit cell subjected to appropriate periodic boundary conditions. Detailed results are provided for the special case of a cubic unit cell with transverse cylindrical voids. The Fe-SMA considered is a $\mathrm{Fe}-\mathrm{Mn}-\mathrm{Si}$ alloy, for which an accurate model was developed recently by the authors and sufficient experimental data is available to allow characterizing the material parameters. 


\section{REVIEW OF CONSTITUTIVE RELATIONS}

The derivation of the constitutive relations, as carried out in (6), gives the following stress-strain relation:

$$
\boldsymbol{\sigma}=\boldsymbol{K}:\left(\boldsymbol{\varepsilon}-\boldsymbol{\varepsilon}^{\mathrm{tr}}-\boldsymbol{\varepsilon}^{\mathrm{pl}}\right)
$$

where $\boldsymbol{\sigma}$ is the stress, $\boldsymbol{\varepsilon}$ is the total strain, $\boldsymbol{\xi}$ is the volume fraction of martensite, $\boldsymbol{\varepsilon}_{\mathrm{tr}}$ is the inelastic phase transformation strain and $\boldsymbol{\varepsilon}^{\mathrm{pl}}$ is the plastic strain. The elastic stiffness tensor $\boldsymbol{K}$ is a function of $\xi$ and is given by

$$
\left[(1-\xi) \boldsymbol{K}_{\mathrm{a}}^{-1}+\xi \boldsymbol{K}_{\mathrm{m}}^{-1}\right]^{-1}
$$

where $\boldsymbol{K}_{\mathrm{m}}$ and $\boldsymbol{K}_{\mathrm{a}}$ are the elastic stiffness tensors of martensite and austenite. Like in most phenomenological models for SMAs, the dissipative state variable $\xi$ is required to satisfy the inequality constraints

$$
\begin{array}{r}
z \geq 0, \\
1-z \geq 0 .
\end{array}
$$

The thermodynamic forces $\mathscr{A}_{\mathrm{tr}}$ and $\mathscr{A}_{\mathrm{pl}}$, conjugate to the dissipative variables $\xi$ and $p$, where $p$ is the magnitude of cumulated plastic strain, are written in terms of the Lagrangian $\mathscr{L}$ for the Fe-SMA, defined in (6), as follows:

$$
\begin{aligned}
& \mathscr{A}_{\mathrm{tr}}=-\frac{\partial \mathscr{L}}{\partial \xi} \\
& \mathscr{A}_{\mathrm{pl}}=-\frac{\partial \mathscr{L}}{\partial p}
\end{aligned}
$$

The loading functions $\mathscr{F}_{\mathrm{m}}^{\mathrm{tr}}$ and $\mathscr{F}_{\mathrm{a}}^{\mathrm{tr}}$, governing the evolution of dissipative variables $\xi$ and $p$, where $p$ is the cumulated plastic strain, are obtained by choosing the conjugate thermodynamic forces $\mathscr{A}_{\mathrm{tr}}$ and $\mathscr{A}_{\mathrm{pl}}$ to be sub-gradients of a dissipative potential $\mathscr{D}(\dot{\xi}, \dot{p})$ given by

$$
\mathscr{D}(\dot{\xi}, \dot{p})=D_{\alpha}^{\mathrm{tr}}|\dot{\xi}|+D_{\mathrm{a}}^{\mathrm{pl}}|\dot{p}|,
$$

where $D_{\mathrm{a}}^{\mathrm{tr}}, D_{\mathrm{m}}^{\mathrm{tr}}$, and $D_{\mathrm{a}}^{\mathrm{pl}}$ are positive coefficient functions of $\xi$, $p$ and the temperature $T$. The detailed expressions of these coefficients can be found in (6). The above gives the following expressions for $\mathscr{F}_{\mathrm{m}}^{\mathrm{tr}}$ and $\mathscr{F}_{\mathrm{a}}^{\mathrm{tr}}$ :

$$
\begin{aligned}
& \mathscr{F}_{\mathrm{m}}^{\mathrm{tr}}=\mathscr{A}_{\mathrm{tr}}-D_{\mathrm{m}}^{\mathrm{tr}}, \text { for forward phase transformation, } \\
& \mathscr{F}_{\mathrm{a}}^{\mathrm{tr}}=-\mathscr{A}_{\mathrm{tr}}-D_{\mathrm{a}}^{\mathrm{tr}}, \text { for reverse phase transformation, } \\
& \mathscr{F}_{\mathrm{a}}^{\mathrm{pl}}=\mathscr{A}_{\mathrm{pl}}-D_{\mathrm{a}}^{\mathrm{pl}}, \text { for plastic deformation. }
\end{aligned}
$$

The flow rules for $\xi$ and $p$ are then given by the Kuhn-Tucker conditions

$\mathscr{F}_{\mathrm{m}}^{\mathrm{tr}} \leq 0, \dot{\xi} \geq 0$ and $\dot{\xi} \mathscr{F}_{\mathrm{m}}^{\mathrm{tr}}=0$ for forward transformation,

$\mathscr{F}_{\mathrm{a}}^{\mathrm{tr}} \leq 0,-\dot{\xi} \geq 0$ and $(-\dot{\xi}) \mathscr{F}_{\mathrm{a}}^{\mathrm{tr}}=0$ for reverse transformation,

$\mathscr{F}_{\mathrm{a}}^{\mathrm{pl}} \leq 0, \dot{p} \geq 0$ and $\dot{p} \dot{\mathscr{F}}_{\mathrm{a}}^{\mathrm{pl}}=0$ for plastic deformation.

The evolution of the transformation strain $\boldsymbol{\varepsilon}^{\text {tr }}$ and the plastic strain $\boldsymbol{\varepsilon}^{\mathrm{pl}}$ are given by

$$
\begin{aligned}
& \dot{\boldsymbol{\varepsilon}}^{\mathrm{tr}}=\dot{\xi} \varepsilon_{\mathrm{L}} \boldsymbol{N}^{\mathrm{tr}}, \text { with } \boldsymbol{N}^{\mathrm{tr}}=\left\{\begin{array}{l}
\frac{3}{2} \frac{V_{\mathrm{tr}}^{\mathrm{dev}}}{V_{\mathrm{tr}}^{\mathrm{eq}}}, \text { if } \dot{\xi}>0, \\
\frac{\boldsymbol{\varepsilon}^{\mathrm{r}}}{\xi}, \text { if } \dot{\xi}<0,
\end{array}\right. \\
& \dot{\boldsymbol{\varepsilon}}^{\mathrm{pl}}=\dot{\xi} \boldsymbol{N}^{\mathrm{pl}}, \text { with } \boldsymbol{N}^{\mathrm{pl}}=\left\{\begin{array}{l}
\frac{3}{2} \frac{V_{\mathrm{pl}}^{\mathrm{dev}}}{V_{\mathrm{pl}}^{\mathrm{eq}}}, \text { if } \sigma_{\mathrm{eq}} \neq 0, \\
\frac{\boldsymbol{\varepsilon}^{\mathrm{pl}}}{\varepsilon_{\mathrm{pl}}^{\mathrm{eq}}}, \text { otherwise. }
\end{array}\right.
\end{aligned}
$$

where $\varepsilon_{\mathrm{L}}$ is the maximum transformation strain and $\boldsymbol{V}_{\alpha}^{\mathrm{dev}}$ and $V_{\alpha}^{\mathrm{eq}}$ are the deviatoric part and the von Mises norm of tensors $\boldsymbol{V}^{\alpha}$, $\alpha \in\{\mathrm{tr}, \mathrm{pl}\}$, which are given by

$$
\begin{aligned}
\boldsymbol{V}^{\mathrm{tr}} & =\frac{1}{\xi} \frac{\partial \mathscr{L}}{\partial \overline{\boldsymbol{\varepsilon}}_{\mathrm{tr}}}, \\
\boldsymbol{V}^{\mathrm{pl}} & =\frac{1}{(1-\xi)} \frac{\partial \mathscr{L}}{\partial \overline{\boldsymbol{\varepsilon}}_{\mathrm{pl}}},
\end{aligned}
$$

where $\mathscr{L}$ is the Lagrangian of the SMA, constructed as the sum of its Helmholtz free energy and energies due to the constraints on the state variables. The overbar in the above equations is used to indicate averaging over a representative volume element.

\section{ALGORITHMIC CONSIDERATIONS AND NUMERICAL SIMULATIONS}

The procedure for discretizing and integrating the constitutive relations in the previous section is detailed in (6), where it is implemented for simulating dense Fe-SMA structures subjected to arbitrary thermomechanical loading. The same model is utilized to investigate, numerically, the effective behavior of FeSMA cells consisting of periodic repetitions of well-defined unit cells. This is illustrated, in this work, by considering the sample unit cell geometry shown in figure 1 . The cell is a cube of $2 \mathrm{~mm}$ side length intersected by two hollow cylinders, each $1.5 \mathrm{~mm}$ in diameter, as shown in the figure. The cell is subjected to periodic boundary conditions in displacement and antiperiodic conditions in traction on opposite faces. These lead, in particular, to the following displacement conditions

$$
u_{j}^{k+}(\boldsymbol{x})-u_{j}^{k-}(\boldsymbol{x})=c_{j}^{k}, \text { with } j, k=\{1,2,3\}
$$




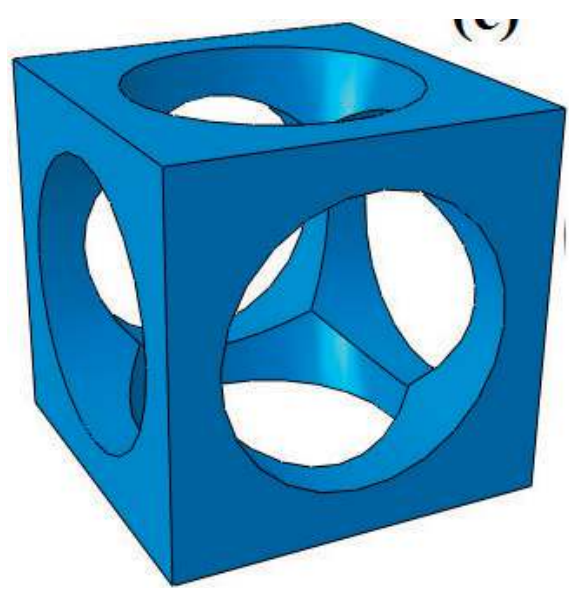

FIGURE 1. Sample unit cell used for finite element simulations using the unit cell method.

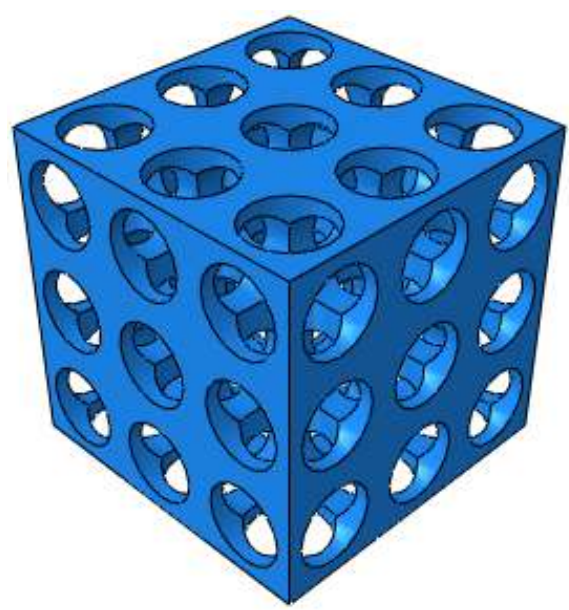

FIGURE 2. Large cell consisting of a periodic assembly of $3 \times 3 \times 3$ unit cells.

following (13), where $u_{j}$ is the $j^{\text {th }}$ component of displacement, $k+$ refers to the face with external unit normal in the positive $x_{k}$ direction and $k-$ to the face with external unit normal in the opposite direction, $c_{j}^{j}$ are constants representing average stretching of the $\mathrm{UC}$, and $c_{j}^{k}=c_{k}^{j}$ represent average shear. The results of numerical simulations for the unit cell are compared to those obtained using the larger cell in figure 2 , which consists of $3 \times 3 \times 3$ cells. In particular, the calculated stress-strain-temperature behavior is shown in figure 3 and the evolution of the martensite volume fraction with the strain and temperature in figure 4.

In both cases, the results are, overall, in agreement, with deviations observed because the larger cell is likely not large enough. Larger cells have not been considered here because of the time required to run the scripted procedure used in extracting data and averaging over the volume of the mesh elements in the large cell.

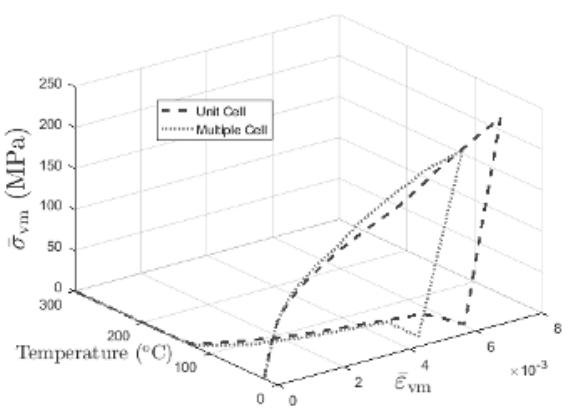

FIGURE 3. Stress-strain-temperature behavior of the unit cell (dashed line) and the $3 \times 3$ cell.

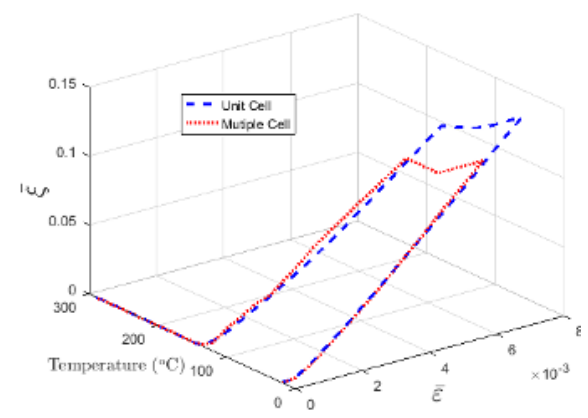

FIGURE 4. Evolution of the volume fraction of martensite in the unit cell (dashed line) and in the $3 \times 3$ cell.

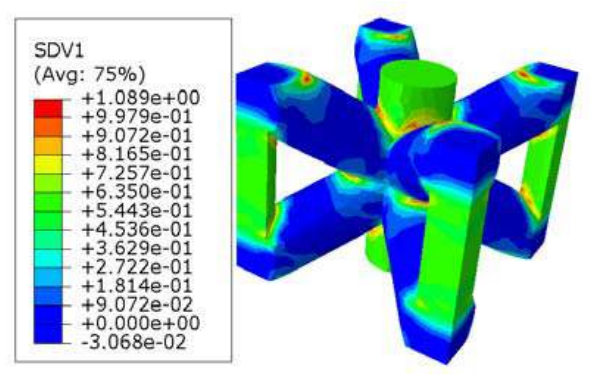

FIGURE 5. Volume fraction of martensite in a unit cell with axial reinforcement subjected to $2 \%$ compression.

Using the unit cell method and the same FEA procedure, other cubic geometries of identical external dimensions and consisting of the same material can be considered. Examples of simulation results for such cells are shown in figures 5 and 6 . The results show the distribution of martensite within the volume of each cell upon compression of $2 \%$ in one of the axial directions starting from an unloaded austenitic state. Values of $\xi$ reported in the legend to be greater than the maximum permissible limit of 1 are due to the way averaging is carried by the FEA software over the mesh elements. No such aberration was observed at the integration points. 


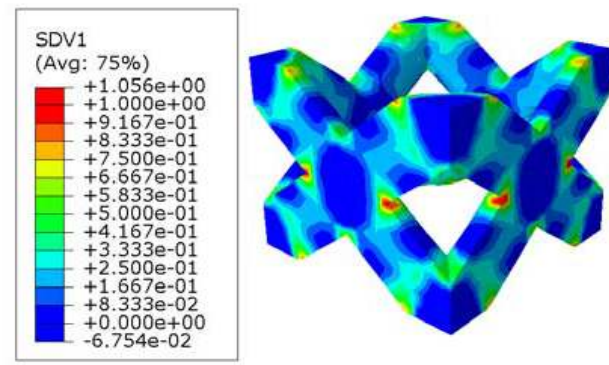

FIGURE 6. Volume fraction of martensite in a unit cell with facial reinforcement subjected to $2 \%$ compression.

\section{CONCLUSION}

Finite element analysis of architectured Fe-SMA samples featuring periodic patterns was carried out in this work. The analysis was simplified by restricting the analysis to unit cells subjected to appropriate periodic boundary conditions. The validity of this approach was validated for one of the geometries considered. The FEA results were used in this case to extract the effective behavior of the architectured material using a volume averaging procedure implemented by means of a script that extracts local mechanical fields in mesh elements and computes averaged values of these fields. The procedure will be utilized in future work for deriving analytical constitutive relations for architectured Fe-SMAs.

\section{ACKNOWLEDGMENT}

Dr. Wael Zaki would like to acknowledge the financial support of Khalifa University through KUIRF level 2 research fund no. 210114.

\section{REFERENCES}

[1] Tanaka, Y., Himuro, Y., Kainuma, R., Sutou, Y., Omori, T., and Ishida, K., 2010. "Ferrous polycrystalline shapememory alloy showing huge superelasticity". Science, 327(5972), pp. 1488-1490.

[2] Wen, Y., Peng, H., Raabe, D., Gutiérrez-Urrutia, I., Chen, J., and Du, Y., 2014. "Large recovery strain in Fe-Mn-Sibased shape memory steels obtained by engineering annealing twin boundaries". Nature communications, 5.

[3] Kajiwara, S., 1999. "Characteristic features of shape memory effect and related transformation behavior in Fe-based alloys". Materials Science and Engineering: A, 273, pp. 67-88.

[4] Cladera, A., Weber, B., Leinenbach, C., Czaderski, C., Shahverdi, M., and Motavalli, M., 2014. "Iron-based shape memory alloys for civil engineering structures: An overview". Construction and Building Materials, 63(0), pp. $281-293$.
[5] Khalil, W., Saint-Sulpice, L., Arbab Chirani, S., Bouby, C., Mikolajczak, A., and Ben Zineb, T., 2013. "Experimental analysis of Fe-based shape memory alloy behavior under thermomechanical cyclic loading". Mechanics of Materials, 63, pp. 1-11.

[6] Cissé, C., Zaki, W., Gu, X., and Zineb, T. B., 2017. "A nonlinear 3D model for iron-based shape memory alloys considering different thermomechanical properties for austenite and martensite and coupling between transformation and plasticity". Mechanics of Materials, 107, pp. 1-21.

[7] Cisse, C., Zaki, W., and Zineb, T., 2016. "A review of modeling techniques for advanced effects in shape memory alloy behavior". Smart Materials and Structures, 25(10).

[8] Cisse, C., Zaki, W., and Ben Zineb, T., 2016. "A review of constitutive models and modeling techniques for shape memory alloys". International Journal of Plasticity, 76, pp. 244-284.

[9] Zaki, W., and Moumni, Z., 2015. "Modeling framework for materials capable of solid-solid phase transformation: Application to the analytical solution of the semi-infinite mode iii crack problem in an idealized shape memory alloy". Vol. 9431.

[10] Moumni, Z., Zaki, W., Nguyen, Q., and Zhang, W., 2015. "Modeling of materials capable of solid-solid phase transformation. application to the analytical solution of the semi-infinite mode iii crack problem in a phase-changing solid”. International Journal of Non-Linear Mechanics, 69, pp. 146-156.

[11] Wang, J., Moumni, Z., Zhang, W., and Zaki, W., 2017. "A thermomechanically coupled finite deformation constitutive model for shape memory alloys based on Hencky strain”. Smart Materials and Structures, 26(6), p. 065006.

[12] Wang, J., Moumni, Z., Zhang, W., Xu, Y., and Zaki, W., 2017. "A 3D finite-strain-based constitutive model for shape memory alloys accounting for thermomechanical coupling and martensite reorientation”. Smart Materials and Structures, 26(6), p. 065006.

[13] Xia, Z., Zhang, Y., and Ellyin, F., 2003. "A unified periodical boundary conditions for representative volume elements of composites and applications". International Journal of Solids and Structures, 40(8), pp. 1907-1921. 